\title{
Quelle place pour les cheveux dans la lutte contre le dopage?
}

\section{Is there a place for hair analysis in doping control?}

Pascal KINTZ

Institut de Médecine Légale, 11, rue Humann - 67000 STRASBOURG - FRANCE Tél : 0388249126 - Fax : 0388356758 - e-mail pascal.kintz@wanadoo.fr

(Reçu le 25 novembre 1999 ; accepté le 18 janvier 2000)

\section{$R E ́ S U M E ́$}

L'analyse des substances de la performance à partir d'une mèche de cheveux permet de mettre en évidence les expositions répétées ou chroniques. En cela, cette approche permet d'augmenter la durée de la fenêtre de détection des substances, communément de l'ordre de 2 à 3 jours, sauf pour les anabolisants sous forme estérifiée, avec l'analyse sanguine ou urinaire.

Les résultats donnent des renseignements sur le profil de consommation pendant plusieurs mois, voire des années, en fonction de la longueur des cheveux, en particulier sur sa sévérité et son évolution. A travers plusieurs exemples dans le suivi des sportifs, la place majeure de l'analyse des cheveux en cette fin de millénaire est illustrée. Cette technologie, acceptée par la plupart des Tribunaux judiciaires mondiaux, n'est pas encore reconnue par le Comité International Olympique. La récente loi du 28 janvier 1999 relative à la protection de la santé des sportifs et à la lutte contre le dopage mentionnant dans son article 14 le contrôle des agissements interdits à partir de "prélèvements biologiques", apparait comme une ouverture à cette approche.
MOTS-CLÉS

cheveux, dopage, analyses.

\section{SUMMARY}

The standard in drug testing for doping control is gas chromatography / mass spectrometry conducted on a urine sample. For the past twenty years, hair analysis has been proposed for identifying drug abusers in forensic science. Specimens can be collected under close supervision without embarrassement and are not subject to evasive maneuvers. In contrast with urine, hair analysis has a wide window of detection, ranging from weeks to months, depending on the length of the hair shaft, and provides information concerning the pattern of an individual's drug abuse. Hair analysis is not yet recognized by the International Olympic Committee, although this technology is accepted in most courts of Justice.

In light of potential applications, hair should be included as suitable specimen to document positive urine cases in doping control.

\section{KEY-WORDS}

Hair, doping control, analysis. 


\section{Consensus de la Society of Hair Testing (1)}

Du 14 au 16 juin 1999, des experts pharmacologues et toxicologues se sont réunis à Martigny (Suisse) pour évaluer les potentialités des cheveux comme milieu d'investigation des substances classées comme dopantes.

Cette manifestation a été organisée par la Society of Hair Testing, société savante créé en décembre 1995 à Strasbourg. Elle regroupe des scientifiques de toutes nationalités, faisant partie de laboratoires de médecine légale, de toxicologie hospitalière, de la police, de la gendarmerie et de l'armée et du secteur privé.

Devant la multiplication des affaires médico-légales concernant des substances augmentant la performance, il a paru important à la Society of Hair Testing de se prononcer sur les applications des cheveux dans l'arsenal analytique de contrôle des pratiques dopantes.

Le consensus ci-dessous est la première étape de réflexion.

Afin d'éviter tout contre-sens, le texte n'a pas été traduit (1). 1. Hair analysis can essentially contribute to doping analysis in special cases, in addition to urine.

2. Hair specimens are not suitable for general routine control.

3. In case of positive urine results, the negative hair result cannot exclude the administration of the detected drug and cannot overrule the positive urine result.

4. In case of negative urine result, the positive hair result demonstrate drug exposure during the period prior to sample collection.

5. Before using hair analysis for doping control, sample collection and analytical methods have to be harmonized with respect to the sophisticated requirements already valid for urine.

6. The Society feels responsible to support efforts that lead to this harmonization.

7. This statment was adopted on June 16, 1999 by the Society of Hair Testing.

\section{Introduction}

Depuis 1968 et les Jeux Olympiques d'hiver à Grenoble, le Comité International Olympique (CIO) et la plupart des fédérations sportives internationales sont concernées par le problème du dopage. Dès le début des contrôles, l'urine a été choisie comme milieu d'investigation. Cette approche, utilisée comme règle de nos jours, permet d'obtenir des informations sur 48 ou 72 heures, exceptionnellement plus, comme pour le cannabis ou les anabolisants sous forme estérifiée. Certaines fédérations sportives autorisent le sang pour évaluer l'hématocrite, paramètre indirect évoquateur d'un abus d'érytropoiétine (EPO), ou, à titre expérimental, l'air expiré pour rechercher les perfluorocarbones (PFC).
A partir de 1979, date des premiers travaux américains (2) et allemands, cette fenêtre de détection a pu être complètement modifiée par l'introduction du cheveu dans l'arsenal analytique. Ce tissu possède la propriété unique d'être le marqueur des expositions répétées ou chroniques, permettant en outre d'établir le profil de consommation à long terme et son évolution. Dans la pratique, l'analyse urinaire et l'analyse des cheveux s'avèrent plutôt complémentaires, les urines permettant de caractériser un usage ponctuel et les cheveux une exposition cumulée $(3,4)$. Le tableau 1 reprend les caractéristiques propres à chaque milieu dans le cadre du contrôle du dopage.

Tableau I: Comparaison des urines et des cheveux dans le cadre des contrôles anti-dopage.

\begin{tabular}{|c|c|c|}
\hline Paramètres & Urines & Cheveux \\
\hline Reconnu CIO & oui & non \\
\hline Substances de la liste & toutes sauf hormones & toutes sauf hormones \\
\hline Dépistage complet & oui & non \\
\hline Techniques analytiques & Immuno, GLC/MS & GLC/MS \\
\hline Fenêtre de détection & $2-5 \mathrm{j}$, sauf anabolisants & plusieurs mois \\
\hline Adultération & possible & très difficile \\
\hline Recueil & invasif & non-invasif \\
\hline Conservation & $+4^{\circ} \mathrm{C}$ ou $-20^{\circ} \mathrm{C}$ & temp. ambiante \\
\hline Analyte majeur & métabolites & substance mère* \\
\hline $\begin{array}{l}\text { Recueil à distance d'un } \\
2^{\text {ence échantillon identique }}\end{array}$ & non & oui \\
\hline Type de mesure & incrémentale & cumulative \\
\hline Risque de faux négatifs & êlevé & faible \\
\hline Risque de faux positifs & théoriquement nul** & théoriquement nul \\
\hline
\end{tabular}

* : en particulier les formes estérifiées, qui ne sont pas d'origine physiologique

** : fonction des seuils retenus par le code médical du CIO

La décennie écoulée a confirmé l'intérêt majeur des cheveux comme marqueurs d'exposition chronique aux xénobiotiques. A présent, les applications de ces investigations débordent du champ purement judiciaire (5-8) dans lequel elles avaient jusqu'alors été confinées, et s'imposent dans un nombre croissant de disciplines cliniques (9).

Par ailleurs, plusieurs affaires judiciaires récentes ont permis de documenter des observations dans le monde du sport, en France, en Allemagne ou encore en Italie. Les cheveux de sportifs ont ainsi été utilisés pour mettre en évidence des pratiques de dopage, des situations de trafic de substances classées comme vénéneuses, généralement des anabolisants ou encore pour infirmer des analyses urinaires contestées (cas le plus fréquent).

Dans sa version définitivement adoptée le 28 janvier 
1999, la loi relative à la protection de la santé des sportifs et à la lutte contre le dopage mentionne dans son article 14 le contrôle des agissements interdits à partir de "prélèvements biologiques", sans préciser la nature de ces prélèvements.

Ainsi, cette revue se propose de résumer les connaissance de l'analyse toxicologique à partir des cheveux et d'évaluer ses applications potentielles dans la lutte contre le dopage.

\section{Incorporation des xénobio- tiques dans les cheveux}

Les poils sont des structures kératinisées propres aux mammifères, produites au niveau d'une invagination de l'épithélium épidermique, le follicule pilo-sébacé. Chacun de ces follicules représente une unité anatomique, constituée du poil proprement dit avec son bulbe pilaire, sa racine et sa tige, du follicule, d'une glande sébacée et d'un muscle horripilateur. L'homme adulte possède environ 5 millions de follicules pileux, dont un million, au niveau du scalp donnent naissance aux cheveux. Une première poussée de poils a lieu vers le 5-6 $6^{\mathrm{mc}}$ mois de la vie fotale : c'est le lanugo. La composition des poils est relativement variable : eau (4-13\%), protéines (85-93\%), lipides (1-3\%) et miné$\operatorname{raux}(0,2-0,8 \%)$.

Les poils se développent puis chutent de façon individuelle et cyclique, selon 3 phases : phase de croissance ou anagène (4 à 8 ans), phase de transition ou catagène ( 2 semaines) et phase de repos ou télogène ( 3 mois). $A$ un instant donné, environ $85 \%$ des cheveux sont en phase anagène. On considère généralement que les cheveux au niveau du vertex poussent de $0,44 \mathrm{~mm} / \mathrm{j}$, soit 1 à $1,3 \mathrm{~cm} /$ mois, avec des variations allant de 0,7 à $1,5 \mathrm{~cm} /$ mois (10).

Sujet de discussion, de controverse, parfois même de polémiques, le mécanisme d'incorporation des xénobiotiques dans les cheveux n'est pas encore bien défini.

Le délai d'apparition d'une substance, défini par le temps écoulé entre l'administration orale ou parentérale et son apparition dans les cheveux, est variable. Selon plusieurs auteurs, il peut être de quelques heures ou de quelques jours. C'est précisément cette différence qui a conduit à envisager 2 modes d'incorporation, l'un rapide et l'autre retardé, correspondant à la durée de l'émergence du poil hors du derme. Ainsi, le mécanisme généralement proposé pour l'incorporation des xénobiotiques dans les cheveux consiste en une diffusion interne des substances du sang vers les cellules en croissance des bulbes pileux et une diffusion externe à partir des secrétions sudorales ou sébacées $(11,12)$.

En fusionnant pour former le cheveu, les cellules en croissance piègeraient les substances dans la structure kératinisée. Les cinétiques d'incorporation sont dépendantes des liaisons du xénobiotique incorporé à la mélanine, un pigment des cheveux. Il semble qu'il existe une différence quantitative d'incorporation suivant la couleur des cheveux, c'est à dire en fonction du degré d'oxydation de la mélanine. Les cheveux foncés, présentant un degré d'oxydation plus important de la mélanine, concentrent ou retiennent plus fortement les drogues que les cheveux clairs, à doses ingérées équivalentes. Cette observation n'est pas sans poser des problèmes d'équité entre les sportifs, puisqu'il est admis par la communauté scientifique qu'à dose équivalente, les concentrations dans les cheveux noirs sont plus importantes que dans les cheveux blonds (12). Ainsi, les cheveux risquent d'être davantage discriminatoire pour les Noirs et donc se posent des problèmes éthniques, voire raciaux, particulièrement sensible aux Etats-Unis. Par ailleurs, il a été observé une nette diminution du contenu en xénobiotiques dans les mèches de cheveux décolorés par rapport aux cheveux de couleur naturelle de la même personne. Cette diminution est de l'ordre de 60 à $70 \%$ pour la cocaïne et ses métabolites et de 70 à $90 \%$ pour les opiacés (13). A ce jour, les décolorations de sportifs sont fréquentes et correspondent à des motifs esthétiques. Néanmoins, elles contribuent à diminuer artificiellement les concentrations, ce qui peut conduire à des résultats faussement négatifs. Chez certains cyclistes avec des cheveux décolorés, il a été difficile de doser la testostérone physiologique, que l'on retrouve à des concentrations inférieures à $1 \mathrm{pg} / \mathrm{mg}$. La liaison à la mélanine ne doit, en fait, représenter qu'une fraction du mécanisme d'incorporation dans les cheveux, car les sujets albinos, dépourvus de mélanine, incorporent tout de même les xénobiotiques.

Les substances mères sont présentes dans les cheveux ou poils à des concentrations plus élevées que celles de leurs métabolites, alors que dans les urines les rapports sont généralement inversés.

L'étroite connexion anatomique entre la tige pilaire et les glandes sudorales et l'analogie des rapports entre molécule mère et métabolites (toujours en faveur de la molécule mère) ont conduit à envisager la sueur comme véhicule d'incorporation des xénobiotiques dans les cheveux. La fixation des xénobiotiques dans les cheveux pourrait également s'effectuer par le biais de l'environnement atmosphérique et concerne plus particulièrement les substances à l'état de particules en suspension. Ainsi, les substances fumées, comme la nicotine, le cannabis, le crack, ou même l'héroïne peuvent se déposer sur toute la longueur du cheveu. Si l'incorporation des xénobiotiques via le sang se fait dans la région médullaire de la matrice kératinisée, dans le cas d'une contamination passive, elle pourrait intervenir plus superficiellement au niveau cortical.

Les substances déposées sur les cheveux par voie pas- 
sive seraient alors moins bien liées à la matrice, ce qui a conduit les toxicologues à développer des méthodes de décontamination des échantillons. Elles consistent en des lavages, soit par une solution aqueuse, soit par un solvant organique, soit par les deux successivement, pendant différents temps d'incubation et à différentes températures. Des cinétiques de lavage et l'analyse des solutions de décontamination ont révélé que les contaminants étaient très vite éliminés (après deux lavages) et qu'ensuite, d'autres lavages n'avaient plus aucun effet.

L'incorporation se faisant dans tous les poils, si les cheveux ne peuvent être prélevés ou sont manquants, d'autres poils conviennent également comme les poils pubiens ou axiliaires. Ces poils sont particulièrement recommandés lorsque les cheveux sont teints ou décolorés.

La stabilité des xénobiotiques une fois incorporés dans les cheveux semble tout à fait exceptionnelle. Il a ainsi été possible d'identifier de la cocaïne dans les cheveux de momies péruviennes, vieilles de plusieurs centaines d'années, prouvant à nouveau l'utilisation de cet alcaloïde par les habitants des Andes.

\section{Prélèvement et analyse}

Les cheveux sont généralement prélevés en vertex postérieur. Une mèche de 60 cheveux (diamètre d'un crayon à papier) est suffisante. Celle-ci doit être prélevée le plus près de la peau, coupée au ciseau (ne pas arracher) et orientée racine extrémité au moyen d'une cordelette, fixée $1 \mathrm{~cm}$ au dessus du niveau de la racine. La conservation est aisée; elle s'effectue en tube sec ou dans une enveloppe, à température ambiante. Ce procédé de stockage est nettement plus favorable que celui des urines qui nécessite le froid.

Le recueil d'un 2ème échantillon identique est toujours possible pour les cheveux alors que cela s'avère irréalisable avec les urines.

De très nombreuses procédures analytiques ont été publiées dans la littérature internationale. La Société Française de Toxicologie Analytique (SFTA) a publié en 1994 un consensus (14) sur l'analyse des opiacés, de la cocaïne et leurs métabolites, basé sur la procédure développée par l'Institut de Médecine Légale de Strasbourg.

A ce jour, parmi les classes de substances interdites et celles soumises à certaines restrictions, les analystes ont pu identifier la plupart des produits, à l'exception majeure des hormones peptidiques et glycoprotéiniques, probablement du fait de leurs encombrements stériques et de leurs poids moléculaires trop importants. Les travaux les plus récents ont essentiellement portés sur la caractérisation des anabolisants (15-24), des ß-adrénergiques (25-28), des dérivés de l'éphedri- ne (29) et des corticostéroïdes $(30,31)$. Toutes les procédures font appel à la spectrométrie de masse, avec filtrage par simple ou triple quadripôle, ou encore par haute résolution. A l'exception des diurétiques et des corticoïdes, identifiés par chromatographie liquide, les autres substances sont toutes séparées par chromatographie en phase gazeuse.

L'acceptation scientifique de l'analyse toxicologique à partir d'échantillons de cheveux est corrélée à la performance des laboratoires pratiquants de telles investigations. Les laboratoires doivent être capable d'identifier les xénobiotiques présents dans les cheveux et d'en mesurer précisément les concentrations. Débutés dès 1992 par le National Institute of Standards and Technologies (USA), les exercices récents ont tous été proposés soit par la Society of Hair Testing (32), soit par la SFTA (33).

Ainsi, plusieurs exercices ont portés sur les opiacés, la cocaïne et ses métabolites, les dérivés de l'amphétamine, les marqueurs du cannabis ou encore les B2-adrénergiques. Ces exercices sont répétés plusieurs fois par an et permettent d'améliorer les procédures analytiques.

\section{Applications}

Que ce soit aux Etats Unis, en Allemagne ou en France, l'expertise toxicologique à partir des cheveux est désormais reconnue par les Tribunaux.

Les cheveux en croissance (environ $85 \%$ de la quantité totale) incorporent les substances présentes dans le sang et la sueur et peuvent ainsi représenter le calendrier rétrospectif de la consommation chronique d'un xénobiotique. En effet, les cheveux poussent d'environ $1 \mathrm{~cm}$ par mois (10) et leur analyse $\mathrm{cm}$ par $\mathrm{cm}$, de la racine (consommation la plus récente) vers la pointe des cheveux (consommation la plus ancienne dans le temps) permet de suivre l'évolution (diminution, augmentation, pas de variation) de la consommation mois après mois.

Aujourd'hui, l'analyse segmentaire est un outil indispensable pour la justice et le corps médical afin de suivre l'évolution d'une toxicomanie ou la substitution par d'autres produits. Néanmoins, les résultats quantitatifs, quels qu'ils soient, doivent être interprétés avec beaucoup de rigueur et de précautions, car si l'analyse segmentaire présente des avantages par rapport aux analyses traditionnelles dans le sang ou les urines (calendrier rétrospectif, fenêtre de détection, évolution de la consommation ...), il faut garder en mémoire que la croissance des cheveux n'est pas continue et que des phénomènes de migration à l'intérieur du cheveu peuvent affecter les concentrations.

Une revue récente (34) a permis de démontrer l'intérêt des cheveux dans le contrôle de l'abus des substances 
de la performance, mais peu de cas sont rapportés dans la littérature internationale. Les résultats les plus probants dans l'identification des substances dopantes dans les cheveux figurent dans le Tableau 2. Gleixner et al (26), en 1996, ont identifié du clenbuterol chez 2 culturistes. Thieme et al $(17,18)$ ont analysé les cheveux d'un culturiste décédé d'un infarctus à 32 ans pour identifier plusieurs esters de testostérone, de la nandrolone décanoate, de la metenolone et de la metandienone. Kintz et al (24) ont retrouvé de la nandrolone, de la testostérone et du stanozolol dans les cheveux de 2 culturistes, accusés de trafic international.

Les concentrations physiologiques de testostérone ont

Tableau II : Compendium de résultats rapportés dans la littérature sur la détection de substances dopantes dans les cheveux.

\begin{tabular}{|c|c|c|}
\hline Références & Substances & Concentrations (pg/mg) \\
\hline 15 & Testostérone* & $1,2-11,4, \mathrm{n}=26$ \\
\hline 20 & Testostérone* & $2,5-4,2, n=6$ \\
\hline 22 & Testostérone* & $3,7-22,4, n=22$ \\
\hline 16 & DHEA** & $0,5-10,6, n=27$ \\
\hline \multirow[t]{6}{*}{17} & Testostérone propionate & 10 \\
\hline & Testostérone isocaproate & 14 \\
\hline & Testostérone phenylpropionate & 28 \\
\hline & Testostérone décanoate & 150 \\
\hline & Nandrolone décanoate & 40 \\
\hline & Metandienone & 58 \\
\hline 18 & Stanozolol & 180 \\
\hline \multirow[t]{3}{*}{21} & Nandrolone & 20 \\
\hline & Methyltestostérone & 170 \\
\hline & Testostérone & $20-150, \mathrm{n}=4$ \\
\hline 23 & Testostérone & 54 et 81 \\
\hline \multirow[t]{3}{*}{24} & Nandrolone & 196 et 260 \\
\hline & Testostérone & 46 et 71 \\
\hline & Stanozolol & 135 et 156 \\
\hline 26 & Clenbuteral & 50 et 92 \\
\hline \multirow[t]{3}{*}{28} & Salbutamol & 71 \\
\hline & Métoprolol & 8410 \\
\hline & Sotalol & 261 \\
\hline \multirow[t]{2}{*}{29} & Ephedrine & $660-10700, n=3$ \\
\hline & Amphétamine & 370 \\
\hline \multirow[t]{6}{*}{35} & Amphétamine & $80-1220, n=10$ \\
\hline & Triamcinolone acétonide & $140-330, n=3$ \\
\hline & Hydrocortisone acétate & 1310 \\
\hline & Methylprednisone & 1210 \\
\hline & Nandrolone & 5,1 \\
\hline & Testostérone décanoate & 15,2 \\
\hline
\end{tabular}

* : concentrations physiologiques chez les hommes

** : concentrations physiologiques chez les hommes et les femmes. été établies par plusieurs équipes $(15,20,22)$ et sont de l'ordre de 1 à $20 \mathrm{pg} / \mathrm{mg}$, pour une moyenne voisine de $3 \mathrm{pg} / \mathrm{mg}$, avec une concentration plus importante chez l'homme que chez la femme. Par contre, il ne semble pas exister de différence liée au sexe en ce qui concerne la dehydroépiandrostérone (DHEA), avec des concentrations variant de 1 à $11 \mathrm{pg} / \mathrm{mg}$ (16).

Idéalement, l'analyse des cheveux autorise la différentiation entre un usage unique et une consommation régulière. De cette façon, des investigations plus poussées auraient permis de confirmer la vague de positifs au cannabis dans le football français.

De même, l'argumentaire d'un surfeur lors des jeux olympique de Nagano sur une contamination passive au cannabis aurait pu être vérifié.

La contamination "accidentelle" ou dans l'intention de nuire d'une boisson par de la cocaïne, par exemple, et qui conduirait à un résultat urinaire positif, pourrait être élucidée par analyse des cheveux, l'athlète pouvant alors prouver une exposition unique.

Dans le cadre de la protection de la santé des sportifs (suivi longitudinal), un usage prolongé de corticostérö̈des ou de B2-adrénergiques ne peut être mis en évidence par analyse urinaire. Au contraire, les cheveux remplissent parfaitement leur rôle de mémoire historique. Les révélations des expertises judiciaires pratiquées après le Tour de France 1998 sont dans ce sens tout à fait éloquentes (35). De même, l'abus d'anabolisants pendant la période hivernale pour augmenter la masse musculaire pourrait être révélé.

Enfin, comme il a été dit précédemment, on retrouve essentiellement la substance mère au niveau des cheveux. Dans le cas d'un abus de dérivés de la testostérone, les cheveux montrent ici un avantage absolu sur les urines. La qualification de dopage se fait sur un rapport testostérone/épitestostérone supérieur à un seuil cible ( 6 ou 10, selon les circonstances). Or, la testostérone est essentiellement administrée par voie injectable, sous forme estérifiée (propionate, énanthate, décanoate ...) qui en se métabolisant va être hydrolysée pour donner de la testostérone, qu'on va retrouver dans les urines en mélange avec la forme endogène. Le dosage urinaire est facile, accessible à de nombreux laboratoires et certains sportifs n'hésitent pas à se faire contrôler régulièrement pour être "en dessous de la barre" ou pour prétexter une secrétion naturelle importante. Dans ces conditions, s'agissant d'un composé physiologique, la différentiation est particulièrement délicate, même si l'utilisation des rapports isotopiques du carbone a été proposée. Au contraire, dans les cheveux, l'ester de testostérone, la substance mère, sera présente et signera de façon indiscutable un apport exogène. Ces analyses sont pratiquées en France et en Allemagne $(15,17,18)$. L'analyse des substances de la performance dans les cheveux se heurte néanmoins à 2 problèmes, leur inef- 
ficacité à déceler les hormones, en particulier l'EPO, et surtout aux problèmes de sensibilité des dosages.

En effet, à ce jour, l'acceptabilité des cheveux est contestée par l'absence d'études contrôlées, en particulier pour les anabolisants, qui sont des substances qui s'incorporent mal, du fait de l'absence d'un atome d'azote dans leur structure, exceptée pour le stanozolol. Dans ces conditions, il peut être difficile de documenter un cas avec des urines positives et des cheveux négatifs si l'expert n'est pas capable de faire la preuve d'une sensibilité analytique suffisante $(19,36)$. Ainsi, il n'a pas été possible d'identifier de la nandrolone après injection intramusculaire de $50 \mathrm{mg}$ de nandrolone undécanoate à un volontaire, alors que ses urines étaient positives pour la norandrostérone et la norétiocholanolone pendant plus de 8 mois (36). Dans ces conditions, l'analyse des cheveux ne peut pas se substituer à un résultat urinaire positif.

Le contrôle direct du dopage par analyse des cheveux ne semble pas être à l'ordre du jour et, dans les faits, ne devrait pas faire l'objet de profondes modifications des règles du code médical du CIO. Par contre, dans le cadre des expertises judiciaires, cette approche, calquée sur les analyses de stupéfiants, peut se révéler déterminante dans la lutte contre la tricherie, la manifestation de la vérité ou les trafics d'anabolisants (37).

\section{Conclusion}

Au total, la demande sans cesse croissante des magistrats d'expertises judiciaires à partir d'échantillons de cheveux a naturellement conduit à standardiser de façon très rigoureuse l'ensemble de la procédure, du prélèvement et de sa conservation à l'interprétation des résultats. Cela implique une chaîne de qualité identique à celle mise en place pour les urines. Chaque laboratoire pratiquant des analyses à partir d'échantillons de cheveux doit avoir une méthodologie complètement validée, incluant précision, justesse, sensibilité et spécificité.

L'analyse des xénobiotiques dans les cheveux semble promise à un bel avenir. A partir d'une standardisation rigoureuse de la méthode de prélèvement et de la technique d'analyse, l'application des cheveux dans la lutte contre le dopage devrait être reconnue.

II n'est certes pas envisageable de rechercher directement l'ensemble des substances classées comme dopantes dans une seule mèche de cheveux. L'urine reste à cet effet le meilleur milieu de dépistage. Mais l'athlète devrait être autorisé à faire pratiquer une analyse de cheveux en cas de contestation, dans des laboratoires reconnus et accrédités par le Ministère de la Jeunesse et des Sports.

\section{Références}

1. Sachs H., Kintz P. Consensus of the Society of Hair Testing on hair testing for doping agents. Forensic Sci. Int. $2000 ; 107: 3$.

2. Baumgartner A.M., Jones P.J., Baumgartner W.A., Black T.C. Radioimmunoassay of hair for determining opiate-abuse histories. J. Nucl. Med. $1979 ; 20$ : 74852 .

3. Du Pont R.L., Baumgartner W.A. Drug testing by urine and hair analysis : complementary features and scientific issues. Forensic Sci. Int. 1995 ; 70 : 63-76.

4. Kintz P. Drug testing in addicts : a comparison between urine, sweat and hair. Ther. Drug Monit. 1996 ; $18: 450-5$

5. Kintz P., Mangin P. Expertises judiciaires à partir d'échantillons de cheveux. J. Med. Leg.. Droit Med. 1995 ; $38: 241-4$.

6. Moeller M.R., Fey P., Sachs H. Hair analysis as an evidence in forensic cases. Forensic Sci. Int. 1993 ; $63: 43-53$

7. Pépin G., Gaillard Y. Applications médico-légales du dépistage dans les cheveux des conduites toxicophiles par chromatographie en phase gazeuse-spectrométrie de masse. Rev. Fr. Lab. 1996 ; 282 : 65-8.
8. Sachs H. Forensic applications of hair analysis. Dans Kintz P., ed. Drug Testing in Hair. 1996 ; Boca Raton : CRC Press;. 211-22.

9. Goullé J.P., Kintz P. Un nouveau moyen d'investigation biologique : l'analyse des cheveux. Intérêt en pratique médicale. Rev. Med. Interne 1996 ; 17 : 826-35

10. Tracqui A. Le poil : structure et physiologie. Rev. Fr. Lab. $1996 ; 282: 19-23$.

11. Cirimele V. Incorporation des xénobiotiques dans les cheveux. Rev. Fr. Lab. 1996 ; 282 : 31-5.

12.Henderson G.L., Harkey M.R., Zhou C., Jones R.T., Jacob P. Incorporation of isotopically labeled cocaine and metabolites into human hair: 1. Dose-response relationships. J. Anal. Toxicol. $1996 ; 20: 1-12$.

13. Cirimele V., Kintz P., Mangin P. Drug concentrations in human hair after bleaching. J. Anal Toxicol. $1995 ; 19: 331-2$.

14.Goullé J.P., Kintz P., Lafargue P., Lardet G., Molinaro R., Pépin G., Tourneau J. Consensus sur l'analyse des substances organiques dans les cheveux. Toxocorama $1994 ; 6,2: 5-8$.

15. Kintz P., Cirimele V., Jeanneau T., Ludes B. Testosterone and testosterone esters identification in human hair. J. Anal. Toxicol. 1999 ; 23 : 352-6. 
16. Kintz P., Cirimele V., Ludes B. Physiological concentrations of DHEA in human hair. J. Anal. Toxicol. 1999 ; 23 : 424-8.

17. Thieme D., Grosse J., Sachs H., Mueller R.K. Detection of several anabolic steroids of abuse in human hair. Dans : Proceedings of the 16th Cologne Workshop on Dope Analysis. 1999 ; Köln : 9-29.

18. Thieme D., Grosse J., Sachs H., Mueller R.K. Analytical strategy for detecting doping agents in hair. Forensic Sci. Int. 2000 ; 107 : 335-45.

19. Segura J., Pichini S., Peng S.H., de la Torre X. Hair analysis and detectability of single dose administration of androgenic steroid esters. Forensic Sci. Int. $2000 ; 107: 347-59$.

20. Scherer C., Wachter U., Wudy S.A. Determination of testosterone in human hair by gas chromatography-selected ion monitoring mass spectrometry. Analyst $1998 ; 123: 2661-3$.

21.Deng X.S., Kurosu A., Pounder D.J. Detection of anabolic steroids in head hair. J. Forensic Sci. 1999 ; 44 : 343-6.

22.Wheeler M.J., ZhongY.B., Kicman A.T., Coutts S.B. The measurement of testosterone in hair. J. Endocrinol. $1998 ; 159$ : R5-8.

23. Höld K.M., Borges C.R., Wilkins D.G., Rollins D.E., Joseph R.E. Detection of nandrolone, testosterone, and their esters in rat and human hair samples. J. Anal. Toxicol. 1999 ; 23 : 416-23.

24. Kintz P., Cirimele V., Sachs H., Jeanneau T., Ludes B. Testing for anabolic steroids in hair from two bodybuilders. Forensic Sci. Int. 1999 ; 101 : 209-16.

25. Gaillard Y., Balland A., Doucet F., Pépin G. Detection of illegal clenbuterol use in calves using hair analysis. Application in meat quality control. J. Chromatogr. B $1997 ; 703$ : 85-95.

26. Gleixner A., Sauerwein H., Meyer H.H.D. Detection of the anabolic B2-adrenoceptor agonist clenbuterol in human scalp hair by HPLC/EIA. Clin. Chem. 1996 ; $42: 1869-71$.
27.Machnik M., Geyer H., Horning S., Breidbach A., Delahaut P., Schänzer W. Long-term detection of clenbuterol in human scalp hair by gas chromatography-high resolution mass spectrometry. J. Chromatogr. B 1999 ; 723 : 147-55.

28. Kintz P., Dumestre-Toulet V., Jamey C., Cirimele V., Ludes B. Doping control for $\beta$-adrenergic compounds through hair analysis. J. Forensic Sci. 2000 ; $45: 170-4$.

29.Dumestre-Toulet V., Kintz P. Ephedrine abuse for doping purpose as demonstrated by hair analysis. J. Anal. Toxicol. $2000 ; 24$ : sous presse.

30. Cirimele V., Tracqui A., Kintz P., Ludes B. First identification of prednisone in human hair by liquid chromatography-ionspray mass spectrometry. J. Anal. Toxicol. $1999 ; 23$ : 225-6.

31. Cirimele V., Kintz P., Dumestre V., Goullé J.P., Ludes B. Identification of ten corticosteroids in human hair by liquid chromatography-ionspray mass spectrometry. Forensic Sci. Int. $2000 ; 107$ : 381-8.

32. Sachs H. Quality control by the society of hair testing. Forensic Sci. Int. $1997 ; 84: 145-50$

33.Deveaux M., Kintz P., Goullé J.P., Bessard J., Pépin G., Gosset D. The hair analysis proficiency testing program of the French Society of Analytical Toxicology. Forensic Sci. Int. 2000 ; 107 :389-94.

34. Kintz P. Hair testing and doping control in sport. Toxicol. Letters $1998 ; 102: 109-13$.

35. Gaillard Y., Vaysette F., Pépin G. Compared interest between hair analysis and urinalysis in doping controls. Results for amphetamines, corticosteroids and anabolic steroids in racing cyclists. Forensic Sci. Int. $2000 ; 107: 361-79$.

36. Kintz P., Cirimele V., Ludes B. Pharmacological criteria that can affect the detection of doping agents in hair. Forensic Sci. Int. 2000 ; 107 : 325-34.

37. Rivier L. Is there a place for hair analysis in doping controls ? Forensic Sci. Int. $2000 ; 107$ : 309-23. 